\title{
Coriolis Coupling Constants and Inertia Defect of Urea
}

\author{
M. Campos-Vallette, C. A. Acevedo-Gonzalez, G. Diaz F.*, and R. Peña C.** \\ Universidad de Chile, Facultad de Ciencias, Departamento de Química, \\ Casilla 653, Santiago, Chile
}

Z. Naturforsch. 40 a, 639-643 (1985); received January 19, 1985

\begin{abstract}
Coriolis coupling constants and the inertia defect for urea have been calculated using force constants obtained by the iterative consistency method. The Coriolis coupling constants are very sensitive to the force constant variation and the value of the inertia defect is calculated to be 0.0119 uma $\AA^{2}$.
\end{abstract}

\section{Introduction}

Several papers dealing with the vibrational spectra and normal coordinate analysis of urea have appeared [1-6]. Duncan [1] computed a 21 parameter symmetry valence force field and Puranik et al. [2] have considered the molecule as a four body problem using a general quadratic potential function. Moreover Annamalai et al. [3] have performed symmetry force field calculations for the planar vibrations of urea using CNDO/Force method and Saito et al. [4] have employed a general valence force field for the non planar ones. It seems, however, that the assignment of the non planar vibrations of urea have not definitely been settled to date. A normal coordinate analysis and the calculation of both Mean Amplitudes and Centrifugal Distortion constants for urea and urea- $\mathrm{d}_{4}$ were performed from a symmetry force field [5]; this study was partially continued for the series including selenourea, thiourea and urea by reporting the force field in internal coordinates for the in plane molecular vibrations, and the corresponding mean amplitudes [6]. In order to complete this study we carried out, for the first time, a normal coordinate analysis in internal coordinates for the out of plane vibrations. This treatment which permits to make a more confidence theoretical assignment using the

* Academia Superior de Ciencias Pedagógicas de Valparaíso, Departamento de Química, Casilla 34-V, Valparaíso, Chile.

** Academia Superior de Ciencias Pedagógicas de Santiago, Departamento de Química, Macul 743, Santiago, Chile.

Request for reprints should be adressed to Prof. Marcelo Campos V., Departamento de Química, Universidad de Chile, Facultad de Ciencias, Casilla 653, Santiago, Chile.
$L$ matrix, allowed the evaluation of Compliance Constants, Coriolis coupling constants and Inertia Defect of Urea.

\section{Experimental}

Commercially available urea was recrystallized several times from ethanol. The purified urea was converted into urea- $\mathrm{d}_{4}$ by the usual exchange reaction with $\mathrm{D}_{2} \mathrm{O}$ obtained from Merck AG, 99.7\% purity. In order to make the deuteration as complete as possible, the exchange reaction was repeated three times. The infrared spectra from 4000 to $250 \mathrm{~cm}^{-1}$ were recorded on a Perkin-Elmer 621 spectrophotometer. The measurement was made for the crystal dispersed in Nujol and $\mathrm{KBr}$ mulls. The spectra are similar to those recorded by Saito et al. [4].

\section{Calculations}

\section{Normal coordinate analysis}

The molecular model and the internal coordinates of urea are shown in Figure 1. The group symmetry is $C_{2 v}$ and the normal modes are distributed in 13 in plane vibrations: $7 \mathrm{~A}_{1}+6 \mathrm{~B}_{1}$; and 5 out of plane vibrations: $3 \mathrm{~B}_{2}+2 \mathrm{~A}_{2}$. The structural parameters were taken from the data of the neutron diffraction analysis of Worsham et al. [7].

\section{a) In plane vibrations}

The normal coordinate analysis for the in plane vibrations of urea and urea- $\mathrm{d}_{4}$ has been carried out using the force field obtained by the iterative con- 


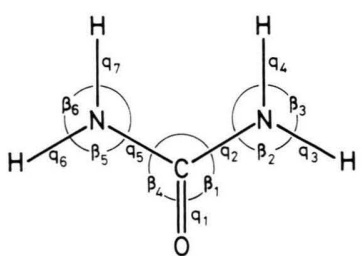

(a)<smiles>NC(N)=O</smiles>

$\theta$<smiles>[B]N([10BH])C(N)=O</smiles>

$\tau$<smiles>NC(=O)NO</smiles>

$\chi$
Fig. 1. Model and internal coordinates of urea, a) in plane vibrations, b) out of plane vibrations [11].

sistency method [8]. The reliability of the force constant results given by this method has been tested in series of simple polyatomic molecules [9]. The in plane $F$ matrix is presented in Table 1. The assignment of the calculated fundamental frequencies was discussed and compared with experimental data in [6].

\section{b) Out of plane vibrations}

The out of plane fundamental frequencies of urea and urea- $\mathrm{d}_{4}$, which are necessary to calculate the force field using the iterative method were selected from our spectra and reported data $[4,5,10]$. The diagonal values of the $F^{0}$ matrix for urea, which allow the initiation of the iterative process, are calculated from the equation $F_{i i}^{0}=\lambda_{i} / G_{i i}$ where $i_{i}=\left(\omega_{i} / 1303.16\right)^{2}$ and $\omega_{i}$ the $i^{\text {th }}$ experimental vibration frequency. The $G$ matrix was constructed by the explicit formulation given by Aroca et al. [11]. The final $F$ matrix obtained after 17 iterative steps is shown in Table 2. The assignment of theoretical and experimental frequencies of urea and urea- $\mathrm{d}_{4}$ made using the $L$ matrix and the Potential Energy Distribution, P.E.D., is given in Table 3.

\section{Compliance constants}

The advantages in the determination of compliance constants, $F^{-1}$, over the more common force constants are well known [12]: the most noteworthy being the simplicity in the exclusion of dependent forces which are conjugate with dependent coordinates. Moreover, the coefficients are determined only by the selection of these forces and are independent of the remainder. This allows the compilation of tables of compliance constants for the structural elements, and simplifies the task of treating molecules with similar structural groups.

The compliance constant matrix for in and out of plane vibrations of urea are displayed in Table 4.

\section{Coriolis coupling constants}

A knowledge of the approximate values of Coriolis coupling constants, $\zeta$, which represents a form of vibration-rotation interaction constants, can be useful for preliminary analysis of rotational structure, prediction of band shapes etc. In calculat-

Table 1. Force constants * for the in plane vibrations of urea.

\begin{tabular}{|c|c|c|c|c|c|c|c|c|c|c|c|c|}
\hline$q_{1}$ & $q_{2}$ & $q_{5}$ & $q_{3}$ & $q_{6}$ & $q_{4}$ & $q_{7}$ & $\beta_{1}$ & $\beta_{4}$ & $\beta_{2}$ & $\beta_{5}$ & $\beta_{3}$ & $\beta_{6}$ \\
\hline 10.7461 & $\begin{array}{l}0.7661 \\
8.0312\end{array}$ & $\begin{array}{l}0.7661 \\
0.7321 \\
8.0312\end{array}$ & $\begin{array}{l}0.0345 \\
0.0913 \\
0.0631 \\
6.4040\end{array}$ & $\begin{array}{l}0.0345 \\
0.0631 \\
0.0913 \\
0.0075 \\
6.4039\end{array}$ & $\begin{array}{r}0.1168 \\
-0.0251 \\
-0.0218 \\
-0.0124 \\
-0.0024 \\
6.3220\end{array}$ & $\begin{array}{r}0.1168 \\
-0.0218 \\
-0.0251 \\
-0.0024 \\
-0.0124 \\
-0.0035 \\
6.3220\end{array}$ & $\begin{array}{r}1.1587 \\
-0.2517 \\
-0.8696 \\
-0.0300 \\
-0.0255 \\
0.0021 \\
0.0243 \\
2.7290\end{array}$ & $\begin{array}{r}1.1587 \\
-0.8693 \\
-0.2517 \\
-0.0255 \\
-0.0300 \\
0.0243 \\
0.0021 \\
1.7152 \\
2.7290\end{array}$ & $\begin{array}{r}-0.0470 \\
0.1387 \\
0.4459 \\
0.0250 \\
0.0451 \\
0.1395 \\
-0.0011 \\
0.0216 \\
0.0514 \\
1.3511\end{array}$ & $\begin{array}{r}-0.0470 \\
0.4459 \\
0.1387 \\
0.0451 \\
0.0250 \\
-0.0011 \\
0.1395 \\
0.0514 \\
0.0216 \\
-0.0522 \\
1.3511\end{array}$ & $\begin{array}{r}0.0260 \\
-0.2163 \\
0.0353 \\
-0.0399 \\
0.0171 \\
-0.1358 \\
-0.0080 \\
0.0183 \\
0.0094 \\
0.4564 \\
-0.0909 \\
0.8269\end{array}$ & $\begin{array}{r}0.0260 \\
0.0353 \\
-0.2163 \\
0.0171 \\
-0.0399 \\
-0.0080 \\
-0.1358 \\
0.0094 \\
0.0183 \\
-0.0909 \\
0.4564 \\
-0.0204 \\
0.8269\end{array}$ \\
\hline
\end{tabular}

\footnotetext{
* Units: stretch: mdyn $\AA^{-1}$; stretch-bend: mdyn; bending: mdyn $\AA$.
} 
ing Coriolis coupling constants, we have used the matrix equation of Meal and Polo [13]

$$
\zeta=L^{-1} C^{\alpha} \tilde{L}^{-1} \text {. }
$$

The elements of the $C^{\alpha}$ matrix were evaluated using standard formulae given by Aroca et al. [11] and Ponomariev [14].

Table 2. Force constants in mdyn $\AA$ for the non planar vibrations of urea.

\begin{tabular}{rrrrr}
\hline$f(\Theta)$ & $f\left(\tau_{1}\right)$ & $f\left(\tau_{2}\right)$ & $f\left(\chi_{1}\right)$ & $f\left(\chi_{2}\right)$ \\
\hline 0.694 & -0.020 & 0.020 & 0.028 & -0.028 \\
& 0.507 & -0.021 & 0.009 & 0.139 \\
& & 0.507 & 0.139 & 0.009 \\
& & & 0.188 & 0.023 \\
& & & & 0.188 \\
\hline
\end{tabular}

Table 3. Out of plane fundamental frequencies $\left(\mathrm{cm}^{-1}\right)$ and assignments of urea and urea- $\mathrm{d}_{4}$.

\begin{tabular}{|c|c|c|c|c|c|c|}
\hline \multirow[b]{2}{*}{ Sym. } & \multicolumn{3}{|c|}{ Urea } & \multicolumn{3}{|c|}{ Urea-d 4} \\
\hline & $v_{\text {exp. }}$ & $v_{\text {cal. }}$ & assign. & $v_{\text {exp. }}$ & $v_{\text {cal. }}$ & assign. \\
\hline$A_{2}$ & $\begin{array}{l}719 \\
555\end{array}$ & $\begin{array}{l}708 \\
546\end{array}$ & $\begin{array}{l}\tau\left(\mathrm{NH}_{2}\right) \\
\chi\left(\mathrm{NH}_{2}\right)\end{array}$ & $\begin{array}{l}517 \\
420\end{array}$ & $\begin{array}{l}511 \\
419\end{array}$ & $\begin{array}{l}\tau\left(\mathrm{ND}_{2}\right) \\
\chi\left(\mathrm{ND}_{2}\right)\end{array}$ \\
\hline$B_{2}$ & $\begin{array}{l}785 \\
719 \\
500\end{array}$ & $\begin{array}{l}780 \\
709 \\
491\end{array}$ & $\begin{array}{l}\Theta(\mathrm{C}=\mathrm{O}) \\
\tau\left(\mathrm{NH}_{2}\right) \\
\chi\left(\mathrm{NH}_{2}\right)\end{array}$ & $\begin{array}{l}776 \\
510 \\
375\end{array}$ & $\begin{array}{l}777 \\
517 \\
372\end{array}$ & $\begin{array}{l}\Theta(\mathrm{C}=\mathrm{O}) \\
\tau\left(\mathrm{ND}_{2}\right) \\
\chi\left(\mathrm{ND}_{2}\right)\end{array}$ \\
\hline
\end{tabular}

Table 5. Coriolis coupling constants of urea.

\begin{tabular}{|c|c|c|c|c|c|}
\hline \multicolumn{4}{|c|}{$\left(A_{1} \times B_{2}\right)$} & \multicolumn{2}{|c|}{$\left(B_{1} \times A_{2}\right)$} \\
\hline $\begin{array}{r}-0.1025 \\
-0.9220 \\
0.0371 \\
0.9136 \\
-0.0004 \\
0.1838 \\
-0.3253\end{array}$ & $\begin{array}{r}-0.2155 \\
-0.6604 \\
-0.6228 \\
-0.1787 \\
0.2507 \\
0.0317 \\
0.2160\end{array}$ & $\begin{array}{r}0.6989 \\
0.2240 \\
-0.4714 \\
0.0838 \\
0.1397 \\
-0.0687 \\
-0.5547\end{array}$ & & $\begin{array}{r}0.1778 \\
0.4533 \\
-0.7401 \\
-0.3162 \\
-0.3907 \\
0.1841\end{array}$ & $\begin{array}{r}0.7321 \\
-0.5497 \\
-0.1040 \\
-0.0802 \\
-0.1156 \\
-0.2733\end{array}$ \\
\hline \multicolumn{6}{|c|}{$\begin{array}{c}\zeta^{Y} \\
\left.A_{1} \times B_{1}\right)\end{array}$} \\
\hline $\begin{array}{r}-0.0031 \\
-0.1679 \\
0.9356 \\
-0.0176 \\
0.2818 \\
-0.1206 \\
-0.0465\end{array}$ & $\begin{array}{r}-0.1545 \\
-0.0064 \\
0.2953 \\
0.0998 \\
-0.7666 \\
0.3357 \\
0.4233\end{array}$ & $\begin{array}{r}-0.8928 \\
0.2079 \\
0.0147 \\
0.2055 \\
0.0234 \\
0.0014 \\
-0.3377\end{array}$ & $\begin{array}{r}-0.2857 \\
0.2884 \\
-0.0092 \\
-0.7000 \\
0.1231 \\
-0.2708 \\
0.5119\end{array}$ & $\begin{array}{r}0.3116 \\
0.8708 \\
0.1867 \\
0.0403 \\
-0.1737 \\
-0.0878 \\
-0.2491\end{array}$ & $\begin{array}{r}0.0210 \\
-0.2213 \\
0.0385 \\
-0.6634 \\
-0.3320 \\
0.1613 \\
-0.6176\end{array}$ \\
\hline \multicolumn{6}{|c|}{$\zeta^{Z}$} \\
\hline $\begin{array}{c}0.5548 \\
-0.6466 \\
0.5933 \\
-0.264 \\
0.1583 \\
-0.0891 \\
-0.2203\end{array}$ & $\begin{array}{r}0.2097 \\
-0.1302 \\
-0.2543 \\
0.1188 \\
-0.2910 \\
0.4475 \\
0.9236\end{array}$ & & $\begin{array}{r}-0.0115 \\
-0.0567 \\
-0.5910 \\
0.7505 \\
-0.1347 \\
0.4650\end{array}$ & $\begin{array}{r}0.3711 \\
0.4753 \\
0.4862 \\
0.3103 \\
-0.4776 \\
-0.3751\end{array}$ & $\begin{array}{l}0.4549 \\
0.4577 \\
0.1685 \\
0.1560 \\
0.5975 \\
0.0828\end{array}$ \\
\hline
\end{tabular}

Table 4. Compliance constants * of urea.

\begin{tabular}{|c|c|c|c|c|c|c|c|c|c|c|c|c|}
\hline$C\left(q_{1}\right)$ & $C\left(q_{2}\right)$ & $C\left(q_{5}\right)$ & $C\left(q_{3}\right)$ & $C\left(q_{6}\right)$ & $C\left(q_{4}\right)$ & $C\left(q_{7}\right)$ & $C\left(\beta_{1}\right)$ & $C\left(\beta_{4}\right)$ & $C\left(\beta_{2}\right)$ & $C\left(\beta_{5}\right)$ & $C\left(\beta_{3}\right)$ & $C\left(\beta_{6}\right)$ \\
\hline 0.102 & $\begin{array}{r}-0.014 \\
0.137\end{array}$ & $\begin{array}{r}-0.014 \\
-0.009 \\
0.138\end{array}$ & $\begin{array}{r}-0.001 \\
-0.001 \\
-0.001 \\
0.156\end{array}$ & $\begin{array}{r}-0.001 \\
-0.001 \\
-0.001 \\
-0.000 \\
0.156\end{array}$ & $\begin{array}{r}-0.002 \\
0.002 \\
0.002 \\
0.001 \\
0.000 \\
0.160\end{array}$ & $\begin{array}{r}-0.002 \\
0.002 \\
0.002 \\
0.000 \\
0.001 \\
0.000 \\
0.160\end{array}$ & $\begin{array}{r}-0.030 \\
-0.024 \\
0.065 \\
0.002 \\
0.001 \\
0.002 \\
-0.000 \\
0.646\end{array}$ & $\begin{array}{r}-0.030 \\
0.065 \\
-0.024 \\
0.001 \\
0.001 \\
-0.000 \\
0.002 \\
-0.395 \\
0.647\end{array}$ & $\begin{array}{r}0.016 \\
-0.029 \\
-0.046 \\
-0.997 \\
-0.004 \\
-0.033 \\
0.001 \\
-0.007 \\
-0.011 \\
0.947\end{array}$ & $\begin{array}{r}0.016 \\
-0.051 \\
-0.034 \\
-0.004 \\
-0.007 \\
0.001 \\
-0.033 \\
-0.033 \\
0.011 \\
-0.012 \\
0.955\end{array}$ & $\begin{array}{r}-0.011 \\
0.052 \\
0.018 \\
0.010 \\
-0.002 \\
0.045 \\
-0.013 \\
-0.013 \\
0.038 \\
-0.541 \\
0.086 \\
1.527\end{array}$ & $\begin{array}{r}-0.014 \\
0.013 \\
0.046 \\
-0.002 \\
0.011 \\
-0.001 \\
0.045 \\
0.035 \\
-0.018 \\
0.087 \\
-0.530 \\
-0.067 \\
1.541\end{array}$ \\
\hline$C(\Theta)$ & $C\left(\tau_{1}\right)$ & $C\left(\tau_{2}\right)$ & $C\left(\chi_{1}\right)$ & $C\left(\chi_{2}\right)$ & & & & & & & & \\
\hline 1.460 & $\begin{array}{r}-0.007 \\
2.488\end{array}$ & $\begin{array}{l}0.007 \\
0.129 \\
2.488\end{array}$ & $\begin{array}{r}-0.255 \\
0.020 \\
-1.860 \\
6.853\end{array}$ & $\begin{array}{r}0.255 \\
1.860 \\
0.020 \\
-0.820 \\
6.853\end{array}$ & & & & & & & & \\
\hline
\end{tabular}

* Units: stretch: $\AA$ mdyn ${ }^{-1}$; stretch-bend: $\operatorname{mdyn}^{-1}$; bending: $\operatorname{mdyn}^{-1} \AA^{-1} . C(Q)$ represents the generalized force conjugated with the corresponding internal coordinate. 
After Jahn's rule [15] for the molecule of urea, we obtain $\zeta^{X}: A_{1} \times B_{2}$ and $B_{1} \times A_{2}, \quad \zeta^{Y}: A_{1} \times B_{1}$ and $\zeta^{Z}: A_{1} \times A_{2}$ and $B_{1} \times B_{2}$. In the $C^{\alpha}$ calculations the $Y$ axis was taken perpendicular to the molecular plane. The $C^{\alpha}$ and $L$ matrix were reduced by symmetry through a transformation $U$ matrix. For the $A_{1}$ and $A_{2}$ species, the coefficient of the transformation $U_{i}$ are the symmetry coordinate $S$ defined by a linear combination of internal coordinates $Q$ :

$$
S\left(Q_{i}\right)=Q_{i} \text { and } S\left(Q_{i}, Q_{j}\right)=2^{-1 / 2}\left(Q_{i}+Q_{j}\right) .
$$

For the asymmetric vibrations $B_{1}$ and $B_{2}$

$$
S\left(Q_{i}, Q_{j}\right)=2^{-1 / 2}\left(Q_{i}-Q_{j}\right) .
$$

The numerical values of the Coriolis constants for urea are given in Table 5. Another set of Coriolis constants was calculated by Díaz [16] who used a symmetry force field.

\section{Inertia defect}

The general formula for the inertial defect is $\Delta=\Delta_{\text {vib }}+\Delta_{\text {cent }}+\Delta_{\text {elec }}$, where $\Delta_{\text {cent }}$ is the contribution due to centrifugal distorsion and $\Delta_{\text {elec }}$ is the electronic contribution [17].

The inertia defect due to vibration-rotation interaction, $\Delta_{\text {vib }}$, which appears to be the major contribution to the total inertia defect in small molecules, can be obtained from the formula developed by Jeyapandian et al. [17]. That equation relates the fundamental vibrational frequencies with the Coriolis coupling constants.

\section{Discussion}

\section{Spectral assignment and force constants for the out of plane vibrations}

In the following discussion we shall use the infrared spectra of urea and urea- $\mathrm{d}_{4}$ published by Saito et al. [4]. Below $800 \mathrm{~cm}^{-1}$, we identify as fundamental, the bands at 785,719 and $500 \mathrm{~cm}^{-1}$ for the urea and 776,510 and $375 \mathrm{~cm}^{-1}$ for the urea$\mathrm{d}_{4}$. The broad band at $510 \mathrm{~cm}^{-1}$ could be composed by two absorptions: $508 \mathrm{~cm}^{-1}$ assigned to $B_{1}$ vibrational species of the skeletal deformation $[6,10]$ and the $517 \mathrm{~cm}^{-1}$, calculated using the product rule, is assigned to an out of plane vibration.

We have ascribed the bands at 785 and $776 \mathrm{~cm}^{-1}$ to $\mathrm{C}=\mathrm{O}$ out of plane bending $\Theta(\mathrm{C}=\mathrm{O})$. The bands at 719 and $500 \mathrm{~cm}^{-1}$ of urea, which shift by effect of deuteration to 510 and $375 \mathrm{~cm}^{-1}$, are assigned to the torsional vibrational modes $\tau\left(\mathrm{NH}_{2}\right)$ and $\chi\left(\mathrm{NH}_{2}\right)$ respectively (see Figure 1). The proposed assignment agrees well with published data in urea $[4,5]$ and acetamide [18].

There is no available data concerning the two out of plane Raman active modes of urea and urea- $\mathrm{d}_{4}$. We have adopted the same frequencies estimated by Saito et al. [4]: 719 and $555 \mathrm{~cm}^{-1}$ for urea and 517 and $420 \mathrm{~cm}^{-1}$ for urea- $\mathrm{d}_{4}$. These frequencies are consistent with the observed diffusion lines in acetamide and its totally deuterated derivatives [18]. We propose the two higher frequencies as due to the torsional modes $\tau\left(\mathrm{NH}_{2}\right)$ and $\tau\left(\mathrm{ND}_{2}\right)$ and the 555 and $420 \mathrm{~cm}^{-1}$ lines to $\chi\left(\mathrm{NH}_{2}\right)$ and $\chi\left(\mathrm{ND}_{2}\right)$ in agreement with the experimental assignment in acetamide and acetamide- $\mathrm{d}_{5}$ [18].

The theoretical force field of urea, displayed in Table 2, allowed us to calculate the frequencies of urea, urea- $\mathrm{d}_{4}$ and its partially deuteroderivatives. The proposed assignment for urea, based on the $L$ matrix and the P.E.D., agree well with available experimental data $[4,6]$ cf. Table 3 . The $L$ matrix gives directly the symmetry of the normal modes.

Because of the difference between both the method of calculations used to determine the Force Field and the coordinate definitions for the out of plane vibrations modes in urea, the symmetrized $F$ matrix, can not be compared with those proposed by Saito et al. [4] and Díaz et al. [5].

The theoretical assignment of the calculated frequencies for the urea conformational isomers and partially deuteroderivatives are available upon request to our laboratory.

\section{Coriolis coupling constants}

The results of the present work and those obtained by G. Díaz [16] follow the sum rules [19] and the upper and lower limits vary between $-1 \leqq \zeta_{i j}^{\alpha} \leqq+1$ for each constant as is shown in Table 5. The numerical differences between the zeta values of both sets is, in several cases, over $100 \%$ of the numerical value. In the case of constants with high numerical values the differences are more than $60 \%$ of the full range of variation. The same conclusion was raised by Aroca et al. [20] when they compared the Coriolis constants of trans-dichloroethylene with the values obtained by Jeyapandian et al. [17] using 
a different force fields. In the present work as well as in that by Aroca et al. [20], the proposed force fields reproduce the experimental frequencies quite well, although the zeta values are substantially different.

\section{Inertia defect}

The vibrational inertia defect calculated through the formula given in [17], using the zeta values obtained in the present work, is 0.0119 uma $\AA^{2}$. This value gives some orientation about the planarity of urea [21]. The reliability of this result can be measured in this moment only by the resemblance between those values calculated by the procedure proposed in this paper and the experimental ones in other molecules [20]. Moreover, preliminary calculations of the total inertia defect in urea [22], using the formulation proposed by Pierce et al. [23], could indicate that the sum of electronic and centrifugal

[1] J. L. Duncan, Spectrochim. Acta 27 A, 1197 (1971).

[2] P. Puranik and L. Sirdeshmikh, Proc. Indian Acad. Sci. 67, 99 (1968).

[3] A. Annamalai, M. Kanakavel, and S. Singh, Proc. Inidan Acad. Sci. 10, 337 (1978).

[4] Y. Saito, K. Machida, and T. Uno, Spectrochim. Acta 27 A, 991 (1971).

[5] G. Díaz F. and M. Campos-Vallette, Spectroscopy Letters 14, 365 (1981).

[6] M. Campos-Vallette and G. Díaz F., Z. Naturforsch. 37 a, 1289 (1982).

[7] J. Worsham Jr., H. Levy, and S. Deterson, Acta Cryst. 10,319 (1957).

[8] Y. N. Panchenko, G. S. Koptev, N. F. Stepanov, and V. M. Tatievskii, Opt. Spect. 25, 350 (1968).

[9] R. Aroca, E. H. Robinson, and T. A. Ford, J. Mol. Struct. 31, 177 (1976).

[10] D. Hadži, J. Kidrič, V. Kneževic, and B. Barlič, Spectrochim. Acta 32 A, 693 (1976).

[11] R. Aroca M., Y. N. Panchenko, and A. S. Makhniov, Chem. Phys. 7, 457 (1975). contributions are not negligeable with respect to the vibrational inertia defect.

\section{Conclusion}

The set of force constants for the out of plane vibrational modes reproduce well the urea and urea- $\mathrm{d}_{4}$ spectra: the calculated frequencies and the assignment proposed on the basis of the $L$ matrix and the P.E.D. are quite similar to the experimental ones.

Since the force constants, in terms of compliance constants for the in plane vibrations, are correlative for the series involving urea, thiourea and selenourea [6] we expect the same tendency to operate for the out of plane vibrations.

The calculated Coriolis coupling constants appear to be very sensitive to the force field approximations.

[12] V. I. Berezin and E. P. Krainov, Opt. Spectr. 19, 950 (1964).

[13] J. H. Meal and S. R. Polo, J. Chem. Phys. 24, 1119 (1956); ibid., 24, 1126 (1956).

[14] Y. I. Ponomariev, Opt. Spectrosk. 18, 87 (1964).

[15] H. A. Jahn, Proc. Roy. Soc. London 168, 469 (1939).

[16] G. Díaz F., unpublished results.

[17] S. Jeyapandian and G. A. Savari Raj., J. Mol. Struct. 14, 17 (1972); ibid. 8, 17 (1971).

[18] T. Uno, K. Machida, and Y. Saito, Spectrochim. Acta 27 A, 833 (1971); ibid. 31 A, 1237 (1975).

[19] L. Nemes, J. Mol. Spectrosc. 28, 59 (1968).

[20] R. Aroca M., J. Anacona, and E. Clavijo, J. Mol. Struct. 27, 49 (1975).

[21] T. Oka and Y. Morino, J. Mol. Spectrosc. 11, 349 (1963).

[22] G. Díaz F., M. Campos-Vallette, and C. A. AcevedoGonzalez, to be published.

[23] L. Pierce, R. Nelson, and C. Thomas, J. Chem. Phys. 43, 3423 (1965). 